\title{
Design and Implementation of Resource Management System for English Assisted Learning
}

\author{
Lulu Zhang \\ Bohai University, Jinzhou, P.R. China \\ luluzhang10@163.com
}

Keywords: English; assisted learning; resource management system; design; implementation

\begin{abstract}
Information technology and network resources are not only the supplement of English education, but also can promote a change in learning style. This article is based on Oracle database management system and .net platform to develop English assisted learning resource management system. First of all, designed database logical structure is composed of a master data table and 7 secondary data table; Secondly, study the.net platform software frame structure; then, design the working process for the users who are teachers with the method of graphic, and illustrate the function for them; Finally, realize the software which take upload resources module as an example. Using the research content of the development of English assisted learning resources management system in this paper, has lots of advantages such as overall function, simple design, standard operation, easy to maintain, easy to use and good portability, etc.
\end{abstract}

\section{Introduction}

In traditional English classroom, teaching has certain limitations such as limited teaching time , boring teaching content, incomplete content, outdated information, passive leaning and teaching according to students' aptitude, which has long plagued English teaching and affected the students' initiative, thus has a poor teaching effect [1]. With the development of information technology and Internet network, fundamental changes have taken place in people's study way. People can choose their own learning style and content that suitable for them according to their own needs. Reasonable use of information technology and network resources can not only be used to the supplement of school English education, the broadening of students' English learning channels, but also can promote a change in student's study approach. Taking advantage of network resources assisted English learning is beneficial to students' autonomous learning because students can grasp the initiative to choose learning content according to their own needs [2]. This paper uses the Oracle database management system and .net platform to design English assisted learning resource management system.

\section{Database Design}

English assisted learning resource management system includes one master data table and 7 secondary data tables, between secondary data table and master data table is "one to many" relation, a record in secondary data table is corresponding to multiple records of master data table, a record of master data table can only be a record for the secondary data table. Design for the logical structure of table is as shown in Table 1. The Oracle database management system has a variety of data types, logical structure design of Table 1 only chose "the ROWID, CHAR, VARCHAR2, NUMBER, DATE, CLOB" six types of data, the ROWID type is used to identify table rows uniquely; CHAR types are used to store a fixed length string; VARCHAR2 types are used to store the variable-length string; The NUMBER type is used to store digital data; The DATE type is used to store date data; CLOB types are used to storing large quantities of characters.

In the logic structure design of Table 1, the field numbers of main data table (resources table) are from 1 to 21. The six secondary data tables, respectively is: the field numbers of "resource type" table are from 31 to 33, the field numbers of "resource sources" table are from 41 to 43, the field numbers of "application for students" table are from 51 and 53, the field number of "upload the 
personnel" table are from 61 to 66, the field number of "resource level" table are from 71 to 73 , the field number of "resource formats" table are from 81 to 83, and the field number of " resources author " table are from 91 to 96.

Table 1. Design on database of English assisted learning resource management system

\begin{tabular}{|c|c|c|c|c|c|}
\hline No & $\begin{array}{l}\text { Name } \\
\text { of Fields }\end{array}$ & $\begin{array}{c}\text { Type and Width } \\
\text { of Fields }\end{array}$ & No & $\begin{array}{c}\text { Name } \\
\text { of Fields }\end{array}$ & $\begin{array}{l}\text { Type and Width } \\
\text { of Fields }\end{array}$ \\
\hline 1 & ResourceCode & ROWID & 41 & SourceCode & CHAR(4) \\
\hline 2 & ResourceName & VARCHAR2(50) & 42 & SourceName & VARCHAR2(30) \\
\hline 3 & TypeCode & CHAR(4) & 43 & SourceDescription & CLOB \\
\hline 4 & SourceCode & CHAR(4) & 51 & IdoneityCode & CHAR(4) \\
\hline 5 & IdoneityCode & CHAR(4) & 52 & StudentCategory & VARCHAR2(30) \\
\hline 6 & StaffCode & CHAR(4) & 53 & CategoryDescription & CLOB \\
\hline 7 & LevelCode & CHAR(4) & 61 & StaffCode & CHAR(4) \\
\hline 8 & FormatCode & CHAR(4) & 62 & StaffName & VARCHAR2(30) \\
\hline 9 & AuthorityCode & CHAR(4) & 63 & StaffDepartment & VARCHAR2(50) \\
\hline 10 & AuthorCode & CHAR(4) & 64 & TechnicalTitles & VARCHAR2(30) \\
\hline 11 & SourceSize & $\operatorname{NUMBER}(6,2)$ & 65 & ResearchDdirection & VARCHAR2(50) \\
\hline 12 & AttachmentsAmount & NUMBER $(4,0)$ & 66 & ContactTelephone & VARCHAR2(50) \\
\hline 13 & BrowseFrequency & NUMBER(10,0) & 71 & LevelCode & CHAR(4) \\
\hline 14 & DownloadFrequency & NUMBER $(10,0)$ & 72 & LevelName & VARCHAR2(30) \\
\hline 15 & MemoryAddress & VARCHAR2(500) & 73 & LevelDescription & CLOB \\
\hline 16 & UploadDate & DATE & 81 & FormatlCode & CHAR(4) \\
\hline 17 & UploadTime & DATE & 82 & FormatName & VARCHAR2(30) \\
\hline 18 & UploadIP & CHAR(15) & 83 & FormatDescription & CLOB \\
\hline 19 & ResourceEvaluation & VARCHAR2(500) & 91 & AuthorCode & CHAR(4) \\
\hline 20 & UseEnvironment & VARCHAR2(500) & 92 & AuthorName & VARCHAR2(30) \\
\hline 21 & UserGuide & CLOB & 93 & AuthorDepartment & VARCHAR2(50) \\
\hline 31 & TypeCode & CHAR(4) & 94 & TechnicalTitles & VARCHAR2(30) \\
\hline 32 & TypeName & VARCHAR2(30) & 95 & GraduateSchool & VARCHAR2(100) \\
\hline 33 & TypeDescription & CLOB & 96 & FinalDegree & VARCHAR2(30) \\
\hline
\end{tabular}

\section{Software Framework Structure}

Software framework is usually done in order to achieve certain industry standards or software component specifications of specific basic task [3]. This system is based on the development of .net platform, which the frame structure is shown in Fig. 1.

As can be seen from the Fig. 1, the .net framework provides four development language, such as Visual Basic, Visual C++, Visual C\# and Visual J\#. It not only can develop the application program of the traditional structure of $\mathrm{C} / \mathrm{S}$, but also can develop structure program for $\mathrm{B} / \mathrm{S}$ which face to Web. The.net Framework includes two main components [4, 5]:

The Common Language Runtime is the basis of the.net Framework, it can be seen as an agent in code execution management, provides the memory management, thread management and core services such as remote processing, and also enforce strict safety of type and other forms of code accuracy which can improve the safety and reliability.

The Common Language Specification (CLS) is required for many application programs which are a basic function of language. CLS rules define a subset of the common type system, namely all applicable to the rules of the common type system suitable for CLS, unless the CLS defines the 
more strict rules. In order to enhance and ensure the language interoperability, CLS defines a set of functions that developers can be sure to use in multiple languages.

\begin{tabular}{|c|c|c|c|c|}
\hline Visual Basic & Visual $\mathrm{C}++$ & Visuc & & Visual J\# \\
\hline \multicolumn{5}{|c|}{ CLS (Common Language Specification) } \\
\hline \multicolumn{3}{|c|}{ ASP.NET Web Forms Web Services } & \multicolumn{2}{|c|}{ Windows Forms } \\
\hline \multicolumn{5}{|c|}{ ADO.NET and XML } \\
\hline \multicolumn{5}{|c|}{ FRC (Framework Class Library) } \\
\hline \multicolumn{5}{|c|}{ CLR (Common Language Runtime) } \\
\hline & OS (Ope & ys & & \\
\hline
\end{tabular}

Fig. 1. .NET Framework architecture

\section{Teachers User Workflow}

The work process of users about teachers is shown in Fig. 2. Description is as follows: after the process start, enter the login screen, enter the user name and password, if the user name and password are correctly then select the corresponding function to operate, or the system asks whether to login or not, if you don't login again, you will log out. Teacher users' selectable features include: the Resource upload, Resource download, Students information management, Students permission the assignment, the Basic information maintenance, etc.

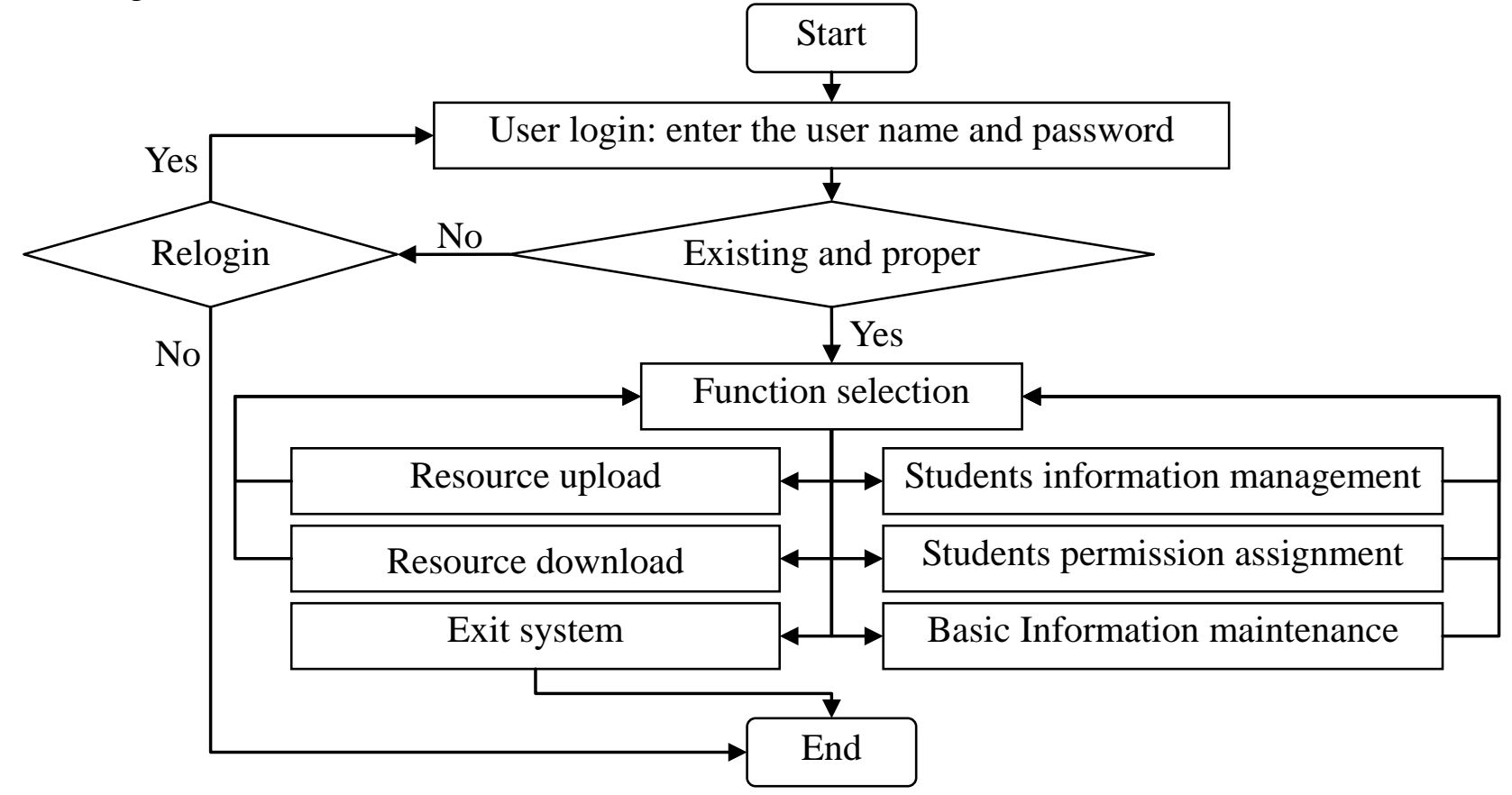

Fig. 2. Teachers user workflow

\section{Design on Resource Upload Module}

Resource upload is the most important function of the users which are teachers, the perform interface is shown in Fig. 3. After entering to uploading interface of English assisted learning resources, the system automatically fills in the "Upload the Date, the Upload Time, Upload IP, Upload People Name, Upload People Code" and other information. Users need to fill out the "Resource Name, Resource Type, Resource Level, the Resource Source, Resource Format, Identity 
Student Resource Author, User Guide" and other information. Use the "Add an Attachment ---" button to add attachments, after adding an attachment, the attachment's name displays in the list box named "Attachments List", the number of "Attachments Amount" control automatically adds 1.

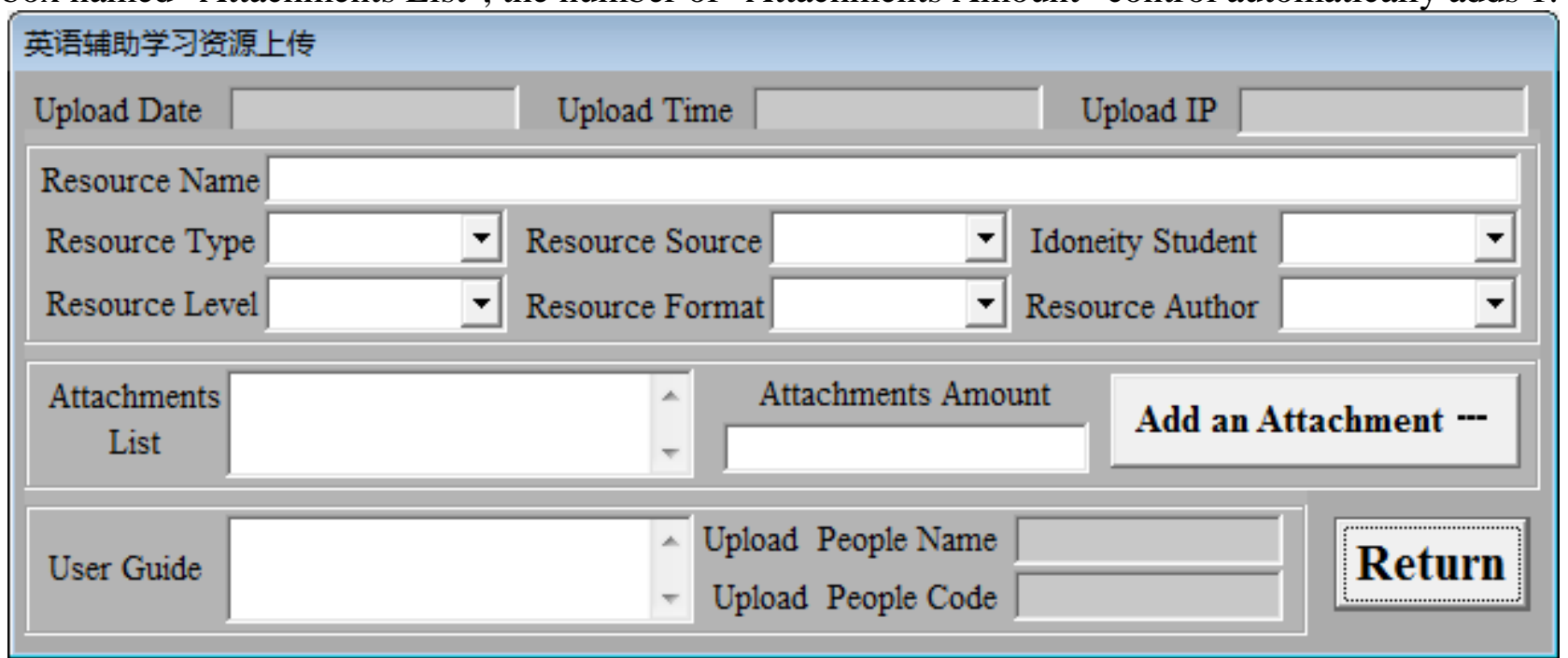

Fig. 3. Upload module on English assisted learning resource

\section{Conclusion}

The online information resources in English can be used in teaching according to teaching materials. By using the sound, images and other information, the aim is to stimulate the students' auditory and visual, arouse the enthusiasm of students' thinking, encourage students' quick perception and strong memory, so as to mobilize the students' observation and imagination, deepen studying impression and improve the learning efficiency. Taking the second language acquisition theory, cognitive theory and constructive theory as the foundation, the English assisted learning resources management system presented in the paper has a quantity of advantages such as fully functional, simple design, standard operation, easy to maintain, easy to use and good portability, etc [6]. The use of the system is of great significance in improving the teaching content, cultivating students' interest in leaning English and enhancing students’ English aptitude, etc.

\section{References}

[1] S. Z. Sun, "Application of computer technology and web resources to assisting English learning," US-China Education Review, vol. 5, no. 4, pp. 24-27, 2008.

[2] M. G. Yan, "Investigation and reflection local college students to use network resources assisted learning English," Journal of Inner Mongolia Normal University(Educational Science), vol. 23, no. 7, pp. 153-156, 2010.

[3] Wikipedia, "Software framework," http://zh.wikipedia.org/wiki/Softwareframework , 2014-9-20.

[4] M. Shi, C. H. Jiang, "Design of Online Business Hall System Based on .NET Platform," Computer Technology and Development, vol. 24, no. 3, pp. 183-186, 2014.

[5] X. W. Ma, L. S. Zhou, X. Cheng, W. Wei, "Information management system for aircraft maintenance based on .NET," Journal of Wuhan University of Science and Technology, vol. 37, no. 1, pp. 75-80, 2014.

[6] J. Zhang, "Exploration on construction and management of university English autonomous learning resources library," Journal of Wuhan Metallurgical Manager’s Institute, vol.23, no. 4, pp. 53-55, 2013. 